\title{
A Case Report of ECT and Muscle Spasms
}

\section{Andia Turner, Matthew Gunther, Majid Husain and Lawrence Faziola*}

Psychiatry and Human Behavior, UCIMC-BLDG 3, Rm 219-RT 88, USA

*Corresponding author: Lawrence Faziola, HS Assistant Clinical Professor, Psychiatry and Human Behavior, UCIMC-BLDG 3, Rm 219-RT 88, USA, Tel: 714 4567304; E-mail: lfaziola@uci.edu

Received date: March 06, 2017; Accepted date: March 25, 2017; Published date: March 31, 2017

Copyright: (C) 2017 Turner A, et al. This is an open-access article distributed under the terms of the Creative Commons Attribution License, which permits unrestricted use, distribution, and reproduction in any medium, provided the original author and source are credited.

\section{Introduction}

Major depressive disorder, a psychiatric condition whereby individuals experience at least one major depressive episode, is a significant health concern in the United States, with the World Health Organization estimating an affected 16 million adults in 2012. Many individuals who seek professional help are successfully treated with pharmacology and/or psychotherapy. For the subset of patients who experience refractory depression, alternative pharmacological treatments are often tried. After a trial of antidepressant medication (lasting at least four weeks and after dose escalation if appropriate) recommendations include atypical antipsychotics, anticonvulsants, and lithium augmentation [1]. Lithium has long been known to be efficacious for the treatment of bipolar disorder, and studies have confirmed its role in unipolar depression, as well as highlighted its anti-suicidal properties [2]. If thorough pharmacological treatment remains ineffective in these patients, Electroconvulsive Therapy (ECT) may be recommended [2]. By nature of this stepwise treatment protocol, a significant number of individuals who require ECT are being prescribed other medications, including lithium. The literature regarding the use of lithium in conjunction with ECT has mixed results, and no clear consensus has been reached [3-5]. This report aims to briefly review the literature regarding lithium use during ECT treatment, as well as present a case of muscle spasms occurring in a patient after receiving short-term lithium treatment with other medications, and co-administered ECT.

Adverse effects have been documented in patients receiving combined lithium and ECT treatment, and include prolonged seizures [3-11] prolonged apnea [5], serotonin syndrome with focal seizures [8], delirium [6,12-15] and declining cognition [9,10]. Other studies have shown no adverse effects. Dolenc and Rasmussen [4] provide a report of 12 cases where ECT and lithium were safely combined without adverse effects. Phase 2 of the PRIDE study showed no remarkable adverse effects for geriatric patients receiving a combination of ECT and a regimen of venlafaxine and lithium [16-20]. A prospective study [12] assessing this combination resulted in no significant differences in seizure variables, apnea time, and recovery from anesthesia. Group differences did exist in autonomic variables: the lithium group showed significantly lower average maximum heart rate and blood pressure than the non-lithium group. This study was the first prospective study to investigate the adverse effects of combining lithium with ECT. Limitations of the study included lack of randomization and a younger patient population (with mean age of 26.00 in the lithium group and 29.78 in the non-lithium group) with no medical comorbidities.

Heinz et al. [13] reported a case where a patient treated with lithium and duloxetine received ECT, and experienced resulting post-ictal ventricular tachycardia. The authors considered a possible interaction between lithium and succinylcholine, and consequently replaced succinylcholine with rocuronium, an alternative muscle relaxant. Additionally, they discontinued duloxetine and reduced lithium. These changes led to the patient experiencing no adverse effects in their next ECT treatment.

The effects of polypharmacy makes the consideration of some of the case reports more complicated as there may be additional mechanism interactions. In the case report by Conway and Nelson [7] describing prolonged seizures during ECT, the patient was being treated with buproprion, venlafaxine, and lithium. A similar article by Rucker and Cook [11] recorded polypharmacy treatment of clomipramine, lithium, 1-tryptophan, quetiapine, and thyroxine leading to prolonged seizures with ECT.

It should also be noted that tardive seizures have been well documented in the literature as occurring after ECT [17-19] although a particular association with lithium has not been made. Tardive seizures after ECT are rare, but potentially dangerous, and have been documented as occurring with a prevalence of $1-2 \%$ per new course of ECT [18]. Tardive seizures occur spontaneously after full recovery from ECT convulsions and are not an extension of the induced seizure. Cases of non-convulsive tardive seizures have also been presented, which may lead to less recognition and treatment, progressing to status epilepticus and associated soft tissue injury, anoxia, and aspiration [17]. Tardive seizures have generally been shown with Electroencephalographic (EEG) evidence of ictal activity [19]. It should be noted that ECT has been successfully repeated after tardive seizures without further complications [17].

\section{Case Description}

A 63-year-old Caucasian man with a diagnosis of major depressive disorder presented to the UC Irvine Medical Center emergency department, after prompting from his outpatient psychiatrist, with Suicidal Ideation (SI) and a plan to shoot himself with a gun. The patient had a 45 -year history of SI and had been battling depression for the majority of his life. His stressors included a car accident six years prior involving his son, in which the son became a quadriplegic after surgery. Additional stressors included his wife's cancer diagnosis and subsequent anxiety over her leaving him as well as struggling with financial issues. This patient had received five previous ECT therapies in the last few weeks at a different facility. These were tolerated well, and he was interested in continuing ECT treatment during an inpatient hospitalization at UCI. At the time of admission into the UCI psychiatry service, the patient was on the following medication regimen: Asenapine $5 \mathrm{mg}$ nightly, trazodone $50 \mathrm{mg}$ nightly, duloxetine $60 \mathrm{mg}$ daily, and buproprion $300 \mathrm{mg}$ in the morning. On admission, he described his mood as "sorrow," and continued to perseverate on his sadness. He denied suicidal ideation in the hospital, but stated that he "might accomplish suicide if outside the hospital." 
The patient's medication regime was slightly modified upon admission: asenapine was discontinued and buproprion was decreased to $150 \mathrm{mg}$ daily. Trazodone and duloxetine were maintained at their stated dosages, and lamotrigine $40 \mathrm{mg}$ daily was started for his mood symptoms. However, the patient continued to report sad mood, anhedonia, guilt, decreased energy and concentration, and suicidal thoughts. Lithium $300 \mathrm{mg}$ three times a day was added one day later to his regimen for mood stabilization and suicide prevention, and then titrated up to $600 \mathrm{mg}$ three times a day. Lamotrigine was discontinued, and Trazadone was increased to $75 \mathrm{mg}$ nightly to improve sleep. Due to the severity of this patient's symptoms, limited response to pharmacological treatment, and previous ECT treatments providing mild relief, a decision was made by the patient and treatment team to proceed with ECT. The patient had been treated with the higher dose of lithium for two days (and treated with lithium overall for six days) prior to ECT. Lithium levels were $0.57 \mathrm{mEq} / \mathrm{L}$ three days prior to ECT treatment, and $0.87 \mathrm{mEq} / \mathrm{L}$ one day prior to treatment, remaining in the therapeutic range.

ECT treatment was initiated on February 9, 2015. The patient received the same doses of medication that had been used for the previous ECT treatments: Brevital $100 \mathrm{mg}$ for induction, succinylcholine $80 \mathrm{mg}$ for muscle relaxation, zofran for nausea, and toradol for post-procedure myalgia. After clinical assessment by the anesthesiologist, an additional $20 \mathrm{mg}$ of Brevital and $20 \mathrm{mg}$ of Succinylcholine were needed based on his response, and these were given prior to treatment initiation. The patient received two right unilateral stimuli due to insufficient duration of the first stimulus. ECT parameters were as follows (with first/second stimuli described respectively): pulse width $0.5 / 0.5 \mathrm{~m} / \mathrm{s}$, frequency $50 / 100 \mathrm{Hertz}$, duration 5.5/5.5 s, and current $800 / 800 \mathrm{~mA}$, energy $29.8 / 52.1$ joules. Total stimuli duration was therapeutic, with motor measuring $15 / 25 \mathrm{~s}$, and EEG measuring $19 / 33$ s. The patient was noted to have dramatic fasciculations as a response to the ECT.

After ECT treatment, the patient complained of painful bilateral muscle spasms in his legs. $1 \mathrm{mg}$ lorazepam was administered, which improved but did not fully resolve his symptoms. He described the spasms as "violent and jerky" movements, paralleling myoclonic jerks. The patient reported that his depressive symptoms remained unchanged after ECT treatment, with continued sadness, anhedonia, decreased concentration/energy, and suicidal thoughts. Given concern that this adverse reaction was linked to his prescribed medications, the patient was presented with the choice to modify his medication regimen (including discontinuation of lithium) and continue ECT treatments, or continue his medication regimen without further ECT. The patient chose to continue his medication regimen without ECT. He continued to receive $1 \mathrm{mg}$ lorazepam every $6 \mathrm{~h}$ and his symptoms significantly improved each day. Mild spasms were still reported four days after ECT, on his day of discharge.

\section{Discussion}

This patient had received five prior ECT treatments without complications, and the only addition with the current ECT experience was the initiation of lithium to his medication regimen. We suspect that an interaction between his medications and ECT led to the muscle spasms. Muscle spasms have not been documented in previous case reports of patients being treated with ECT. It is unclear whether this reaction is: (a) An interaction between lithium and ECT, (b) An interaction between other medications and ECT, or (c) A combination of multiple factors. Multiple possible explanations will be discussed below.

This patient may have experienced a mild prolonged seizure, given the improvement with the anti-seizure medication lorazepam, as well as previously documented prolonged seizure side effects from combining lithium with ECT treatment $[3,6-8,11]$. Also, this patient had been receiving polypharmacy medication, including trazadone, duloxetine, buproprion, and lithium prior to ECT treatment. In the report by Conway and Nelson [7], the combination of lithium with buproprion and venlafaxine (in the same class as duloxetine) resulted in prolonged seizure activity. One of these medications or a combination of them together, may have led to the muscle spasms. Muscle pain is known to be a possible side effect of buproprion and trazodone, and thus these medications may be contributing to the patient's experience.

It is unlikely, though possible, that this patient's muscle spasms were a result of tardive seizure. EEG was not performed and therefore ictal activity cannot definitively be ruled out. This patient had no other signs of abnormal neurological activity and no true ictal or post-ictal state. Also, tardive seizures have generally been reported during a new course of ECT [18] and this patient had previously received five ECT treatments. The only change with this treatment was the initiation of lithium, and there has been no documented association of tardive seizures with lithium use in ECT.

Literature describing an interaction between lithium and succinylcholine has been in the context of prolonged apnea $[5,12,15]$ which this patient did not experience. However, Lithium's interaction with the Neuromuscular blocking agents is well known in the Anesthesia literature. Muscle relaxation for ECT is achieved with a small dose of succinylcholine, a depolarizing blocking agent, and Lithium can potentiate its action and prolong the neuromuscular blocking activity. One of several complications, or adverse effects, of Succinylcholine is fasciculation's leading to myalgia's. This may have also been a contributing factor to the painful and sustained calf muscle pain.

The American Psychiatric Association (APA) task force of 2001 recommends discontinuing lithium or lowering the dosage when combining treatment with ECT [14]. However, the British guidelines of 2006 support the use of lithium when combined with ECT. Based on both recommendations, along with the controversy in the literature and case reports, we recommend that the decision to proceed with ECT while on lithium (or other medications) be based on clinical judgment, taking into consideration each patient's individual condition, as well as the risk-benefit ratio for discontinuing medications and/or ECT treatment. We also feel that patients must be closely monitored for adverse effects after treatment, and it is best to maintain lithium at the lowest effective blood level, and other medications at the lowest effective dose.

The literature would benefit from additional prospective, randomized, clinical trials, without polypharmacy, to better elucidate the interaction and side effect profile of combining lithium and other medications with ECT treatments. Given the practical and ethical challenges in conducting this type of study, especially in such severely refractory patients requiring ECT treatment, the case reports of adverse effects are appreciated and continue to contribute to our collective understanding of this combination treatment. 
Citation: Turner A, Gunther M, Husain M, Faziola L (2017) A Case Report of ECT and Muscle Spasms. J Pharmacovigil 5: 226. doi: 10.4172/2329-6887.1000226

Page 3 of 3

\section{References}

1. Bschor T, Bauer M, Adli M (2014) Chronic and treatment resistant depression: diagnosis and stepwise therapy. Dtsch Arztebl Int 111: 766-776.

2. Bschor $\mathrm{T}$ (2014) Lithium in the treatment of major depressive disorder Drugs 74: 855-862.

3. Sabagh DP, Bijan I, Longshore T (2013) Lithium and Electroconvulsive Therapy: A Case Report. Psychiatric Annals 43: 248-251.

4. Dolenc TJ, Rasmussen KG (2005) The safety of electroconvulsive therapy and lithium combination: a case series and review of the literature. J ECT 21: $165-170$.

5. Hill GE, Wong KC, Hodges MR (1976) Potentiation of succinylcholine neuromuscular blockage by lithium carbonate. Anesthesiology 44: 439-442.

6. Weiner RD, Whanger AD, Erwin CW, Wilson WP (1980) Prolonged confusional state and EEG seizure activity following concurrent ECT and lithium use. Am J Psychiatry 137: 1452-1453.

7. Conway CF, Nelson LA (2001) The combined use of buproprion, lithium, and venlafaxine during ECT: a case of prolonged seizure activity. J ECT 17: 216-218.

8. Sartorius A, Wolf J, Henn FA (2005) Lithium and ECT-Concurrent use still demands attention: three case reports. World J Biol Psychiatry 6: 121-124.

9. Small JG, Kellams JJ, Milstein V, Small IF (1980) Complications with electroconvulsive treatment combined with lithium. Biol Psychiatry 15: 103-112.

10. Milstein V, Small JG (1988) Problems with lithium combined with ECT Am J Psychiatry 145: 1178.
11. Rucker J, Cook M (2008) A case of prolonged seizure after ECT in a patient treated with clomipramine, lithium, l-tryptophan, quetipaine, and thyroxine for major depression. J ECT 24: 272-274.

12. Thirthalli J, Harish T, Gangadhar BN (2011) A prospective comparative study of interaction between lithium and modified electroconvulsive therapy. World J Bio Psychiatry 12: 149-155.

13. Heinz B, Lorenzo P, Markus R, Holger H, Beatrix R, et al. (2013) Postictal ventricular tachycardia after electroconvulsant therapy treatment associated with a lithium-duloxetine combination. J ECT 29: e33-e35.

14. American Psychiatric Association (APA) (2001) The practice of electroconvulsant therapy: Recommendations for treatment, training, and privileging: A task force report of the American Psychiatric Association Washington, DC: American Psychiatric Association Press.

15. Naguib M, Koorn R (2002) Interactions between psychotropics, anaesthetics and electroconvulsive therapy: implications for drug choice and patient management. CNS Drugs 16: 229-247.

16. World Health Organizaton (2015) "Major Depression among Adults." NIMH RSS. National Institute of Medical Health.

17. Felkel WC, Wagner G, Kimball J, Rosenquist P, McCall V, et al. (2012) Tardive Seizure with Postictal Aphasia: A Case Report. J ECT 28: 180-182.

18. Whittaker R, Scott A, Gardner M (2007) The prevalence of prolonged cerebral seizures at the first treatment in a course of electroconvulsive therapy. J ECT 23: 11-13.

19. Thisayakorn P, Karim Y, Yamada T, McCormick LM (2014) A case of atypical tardive seizure activity during an initial ECT titration series. J ECT 30: 77-80.

20. Kellner CH, Husain MM, Knapp RG, Mccall WV, Petrides G, et al. (2016) A Novel Strategy for Continuation ECT in Geriatric Depression: Phase 2 of the PRIDE Study. Am J Psychiat 173: 1110-1118. 